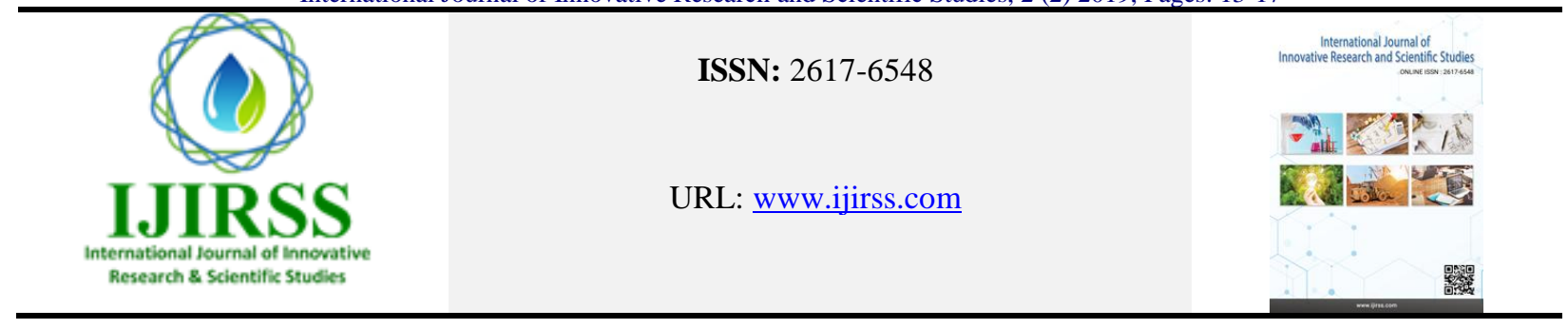

\title{
A Brief Look at the Flow of Mystical thoughts in Turkish Literature and Mention of the Famous Sufis of this Literature
}

\author{
Sima Farda \\ ${ }^{I}$ Department of Uzbek language and literature, Faculty of Education, Jawzjan University, Jawzjan, Aafghanistan \\ (Email: simafarda.2010@gmail.com)
}

\begin{abstract}
To date, the contribution of the Turkish Sufis in the expansion of civilization, wisdom and Sufism are so great and effective in the Islamic world. In this area, many leaders in the golden banners of history have come up with a suitable place for themselves. It should be noted that the evaluation and study of the concepts such as Sufi and Sufism is one of the topics where all their profound and profound learning is essential for any literary worker, and they must investigate it, as it is one of their fundamental duties. The prominent poets of the Uzbek literature such as Khawajah Ahmad Yusawi, Baba Rahim Moshreb, Ibrahim Adham, Yusuf Amdani and others, have left valuable, influential and attractive works in the history of literature of this language, they also uncovered the imperfections of feudalism, the actions of religious hypocrites and unpleasant characteristics of vile leaders. They had a graceful role in deepening of world literature from the viewpoint of language formation and enriching. They have also played a significant role in the use of literary language, instead of colloquial language beside in the improvement of expression technique in this literature. Besides, they had a graceful role in expansion of Uzbek classical literature. The purpose of this article is to motivate learners of Uzbek language and literature in relation with the identification of mystical literature and poets, as well as their role in developing their thoughts and ideas in the right direction. The significance of this paper is to strengthen the worldly and eternal thoughts of peoples and the development of love, affection, intimacy, brotherhood and humanity that lies within their nature and character.
\end{abstract}

Keywords: Religion, Islam, Sufism, Sufi, doctrine, Sheikh, Helal.

DOI: $10.53894 /$ ijirss.v2i2.15

Funding: This study received no specific financial support.

History: Received: 7 April 2019/Revised: 24 April 2019/Accepted: 13 May 2019/Published: 20 May 2019

Licensed: This work is licensed under a Creative Commons Attribution 4.0 License (oc) Ex

Competing Interests: The author declares that there are no conflicts of interests regarding the publication of this paper.

Transparency: The author confirms that the manuscript is an honest, accurate, and transparent account of the study was reported; that no vital features of the study have been omitted; and that any discrepancies from the study as planned have been explained.

Ethical: This study follows all ethical practices during writing. 


\title{
نكاهى مختصر به جريان انديشه هاى تصوفى در ادبيات توركى و ذكر مختصرى از صوفيان مشهور اين ادبيات
}

\author{
سيما فردا \\ دبيارِتـــنت زبان وادبيــات /وزبيــكى، دانشكده تعليم و تربيه، دانشكاه جوزجان، جوزجان، افغانستان \\ خلاصه
}

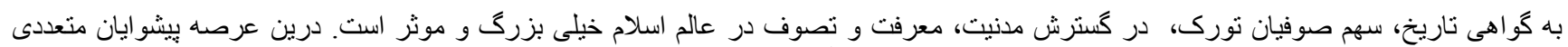

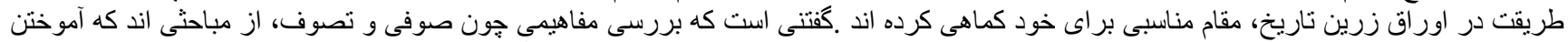

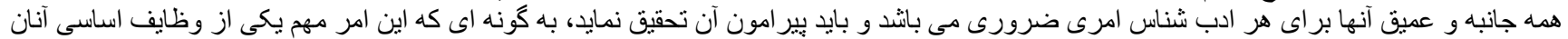

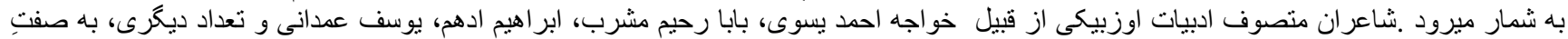

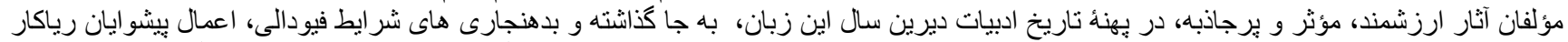

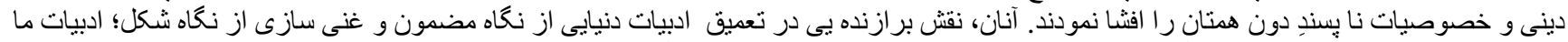

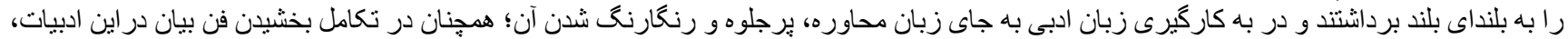

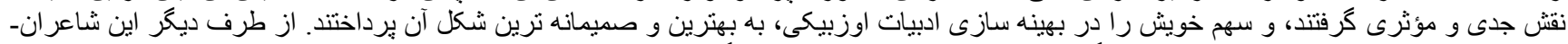

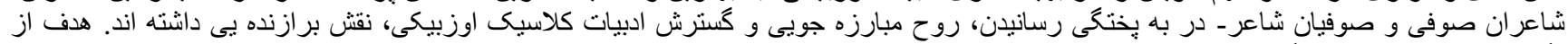

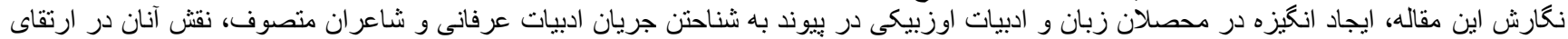

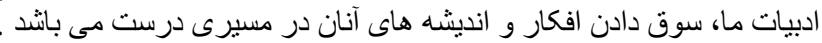

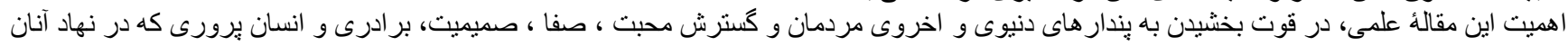
نهفته است

كلمات كليبى: دين، اسلام، تصوف، صوفى، طريقت، عرفان، شيخ، حلال

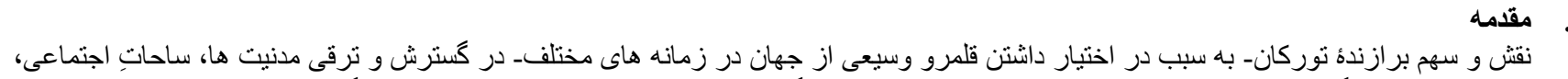

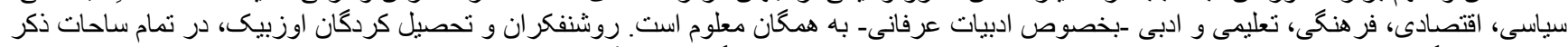

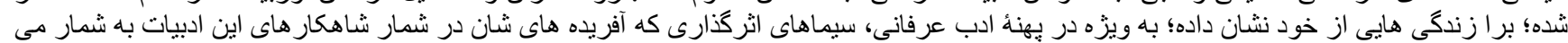

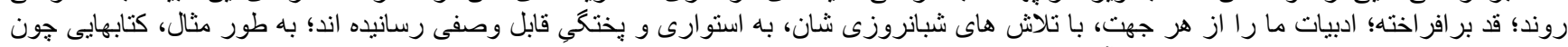

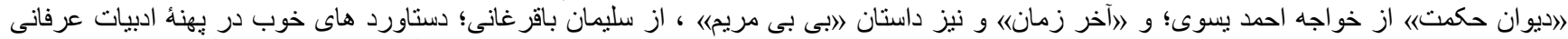

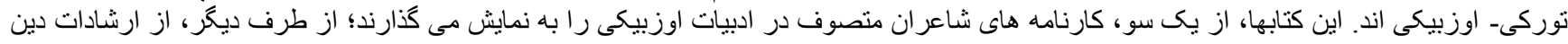

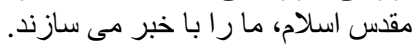

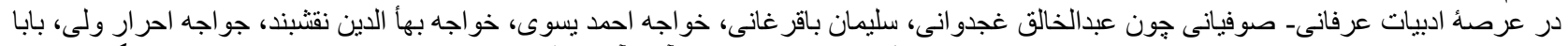

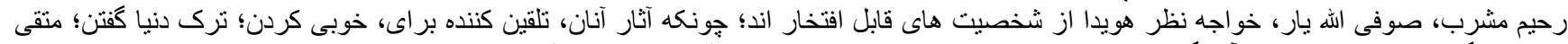

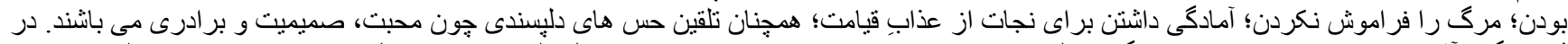

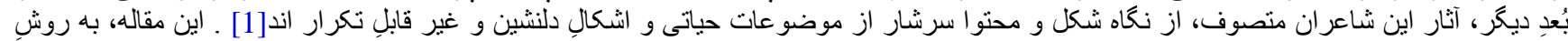

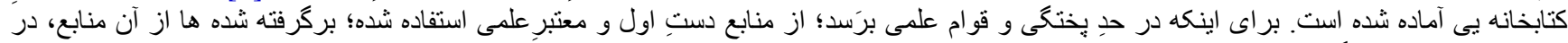
كمال امانتدارى، حفظ كر ديده اند.

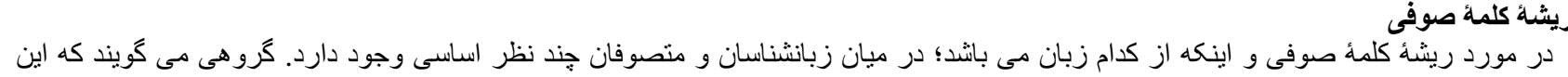

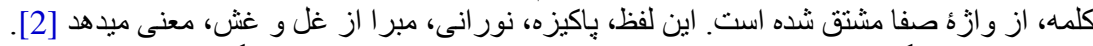

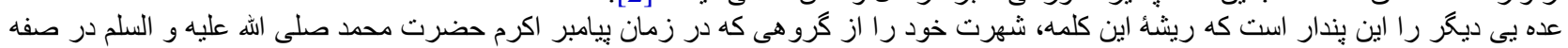

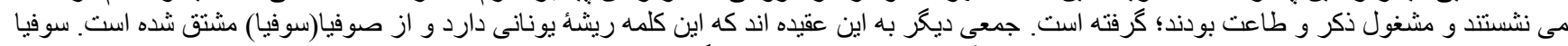

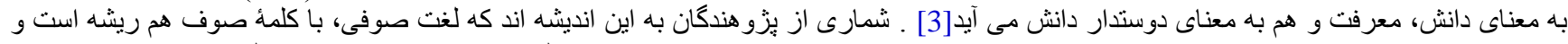

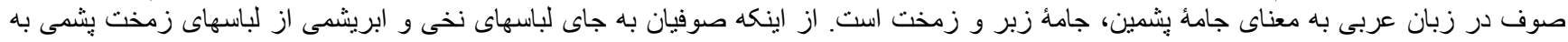

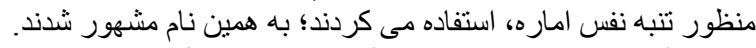

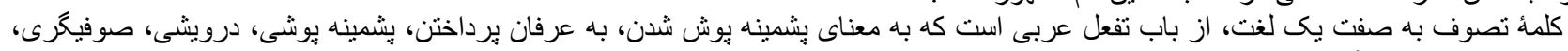

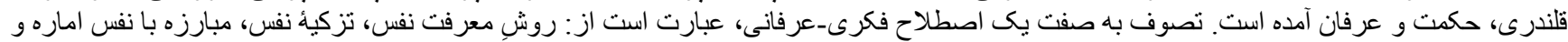

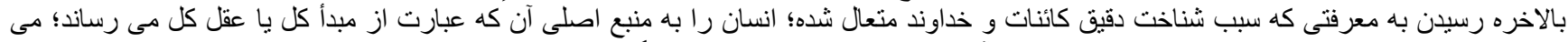

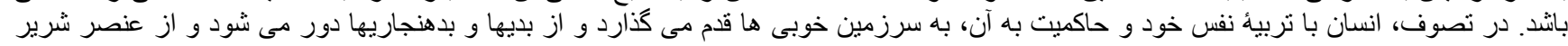

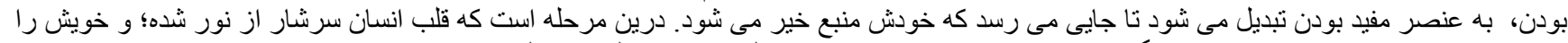

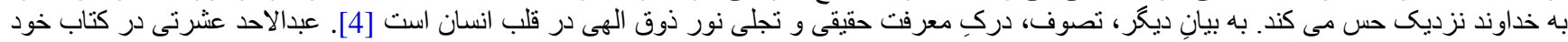

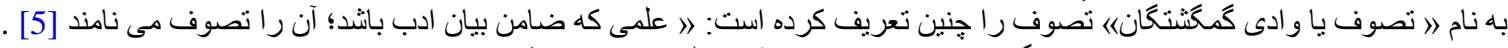

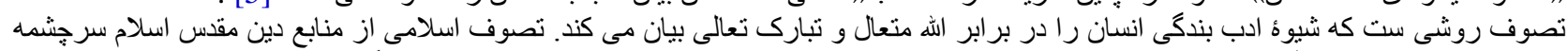

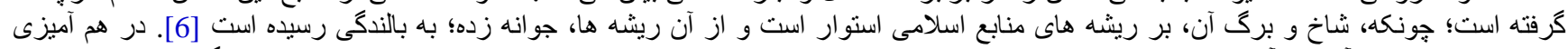

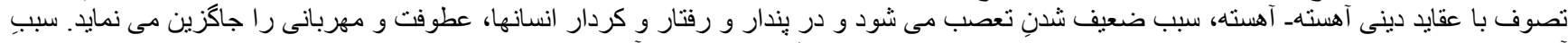

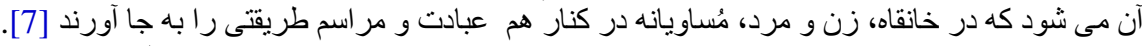

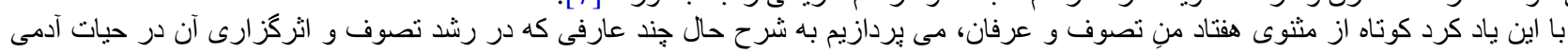

نقشى مؤثر داشته اند. 


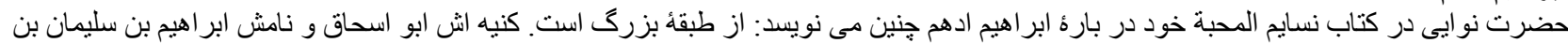

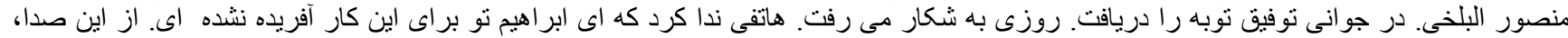

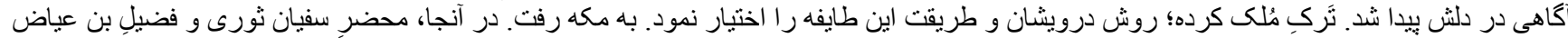

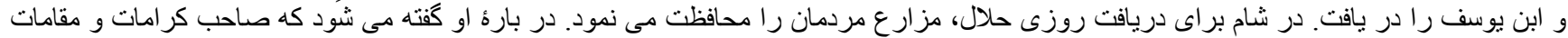

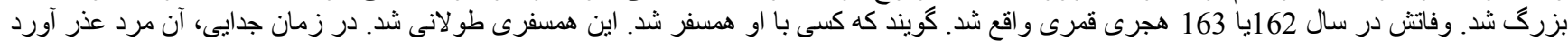

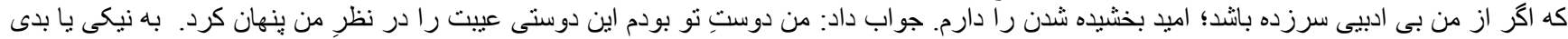
اعمالت نيانديشيدم.

وربيت:

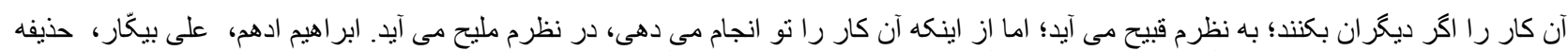

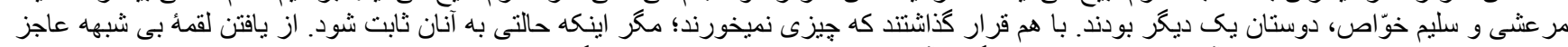

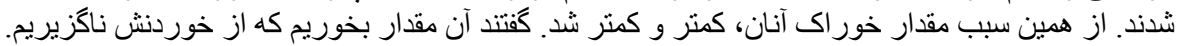

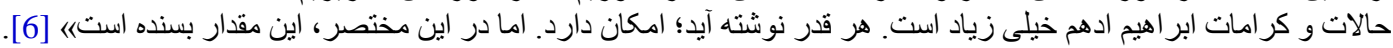

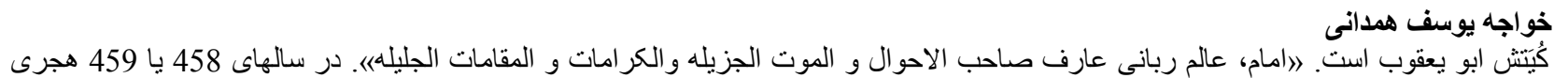

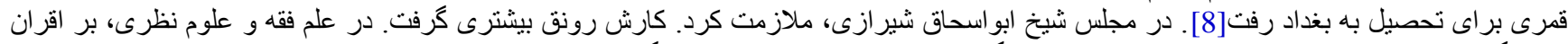

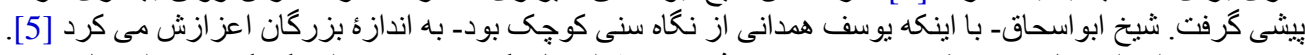

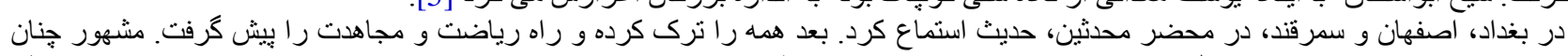

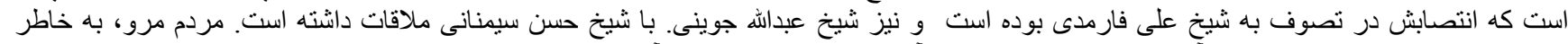

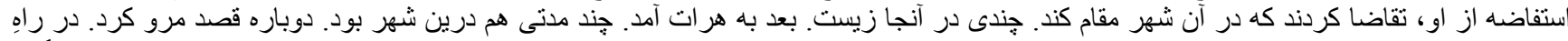

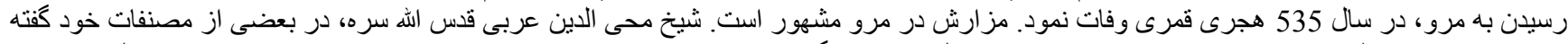

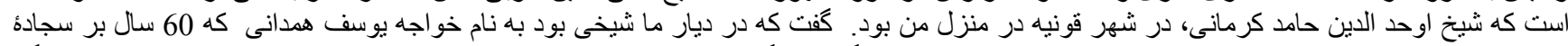

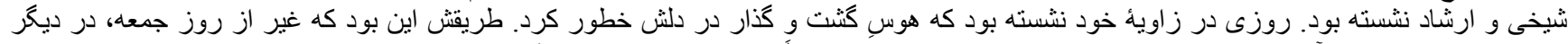

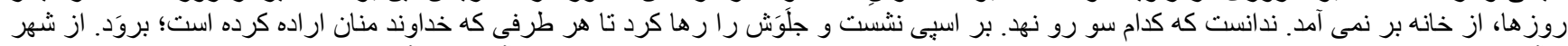

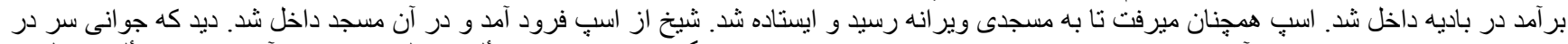

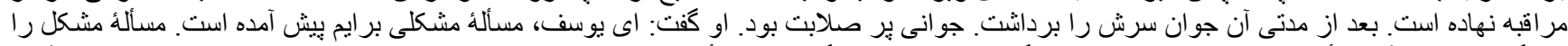

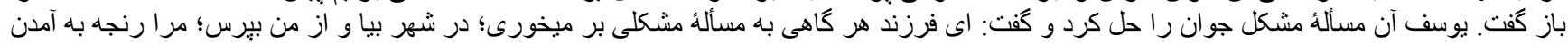

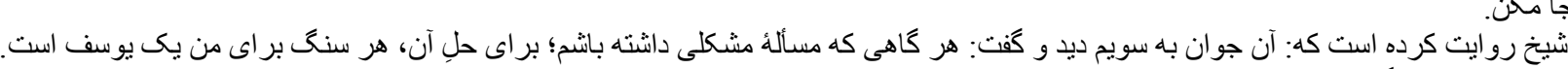

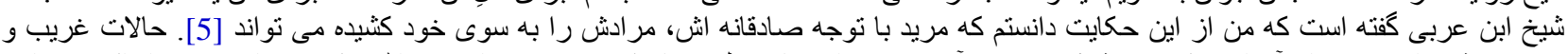

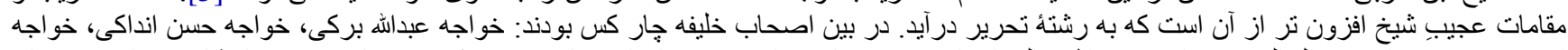

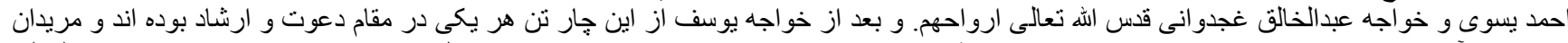

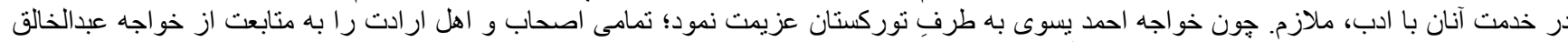

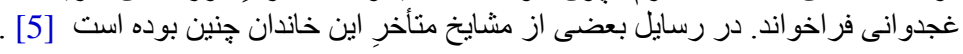

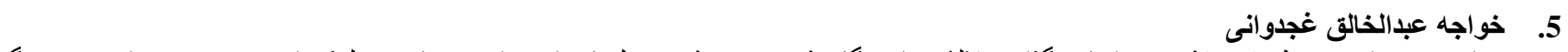

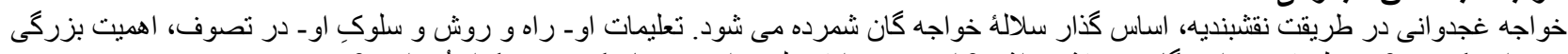

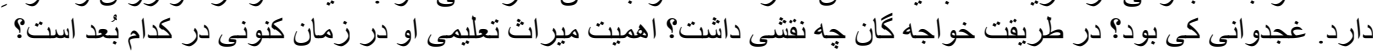

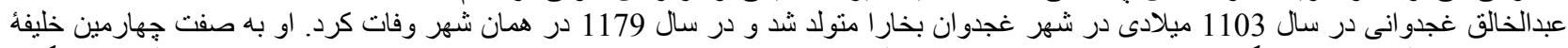

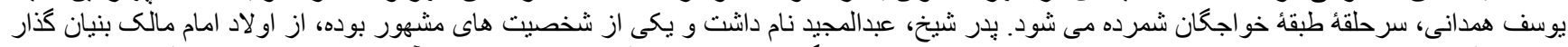

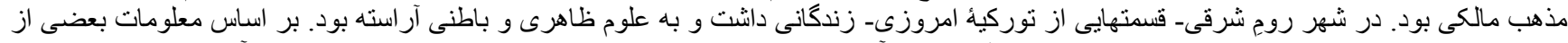

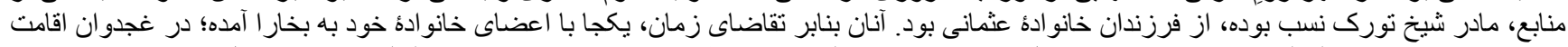

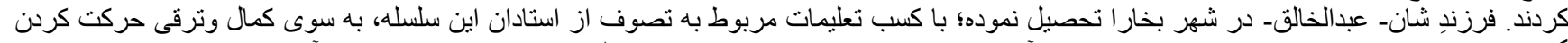

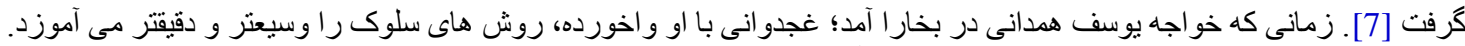

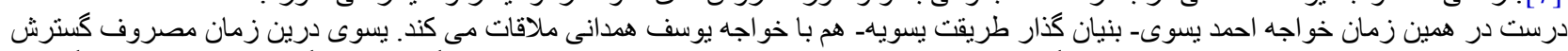

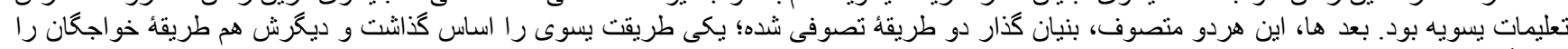

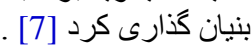

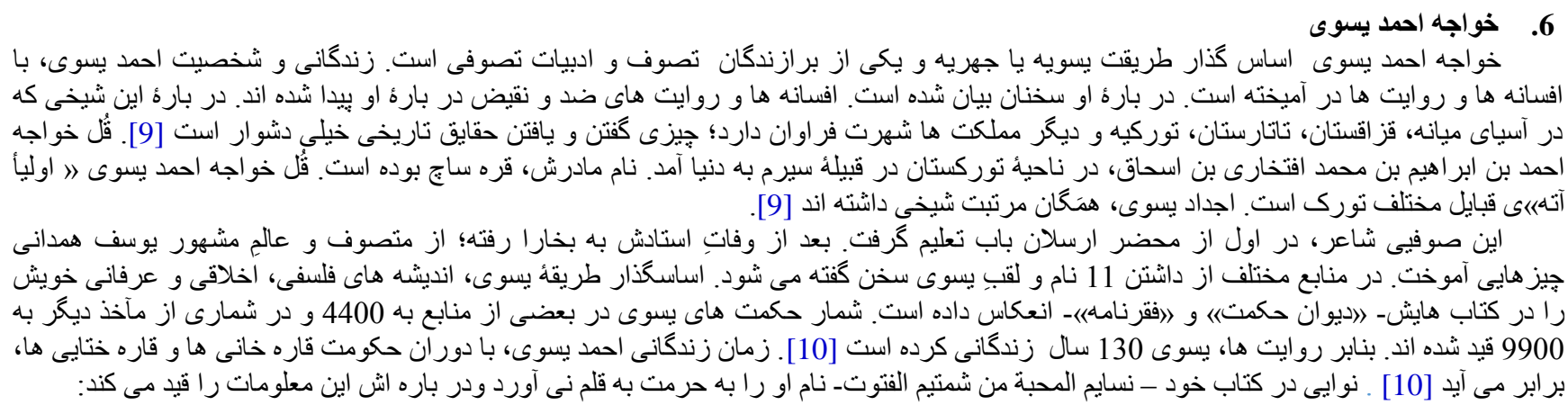




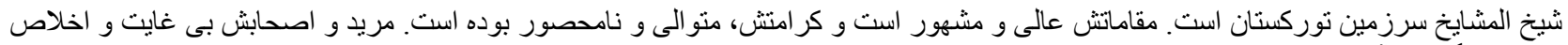

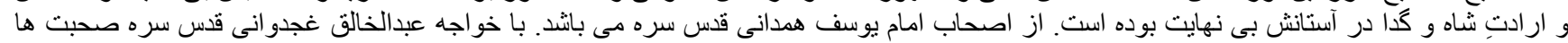

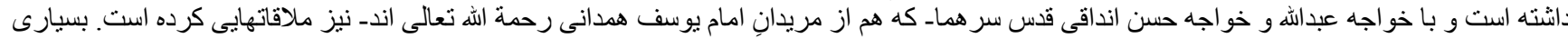

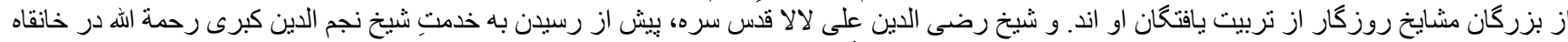

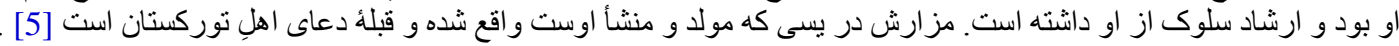

7.

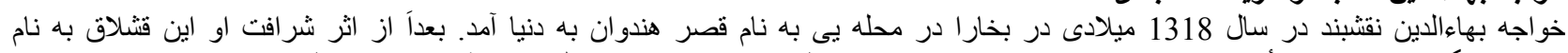

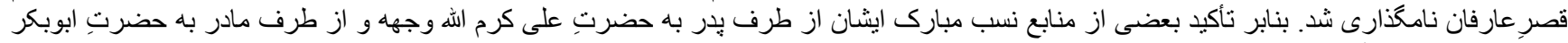

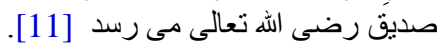

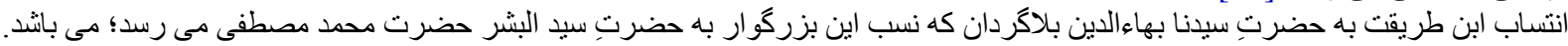

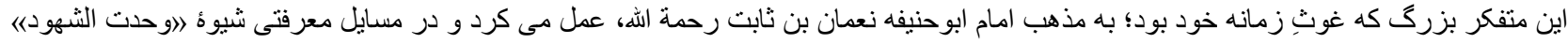

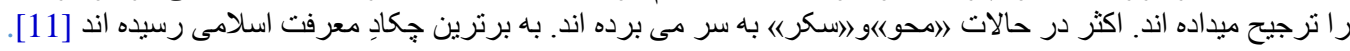

8.

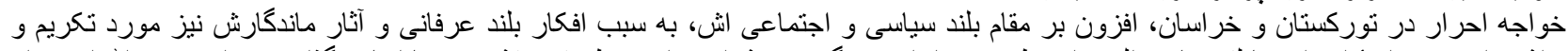

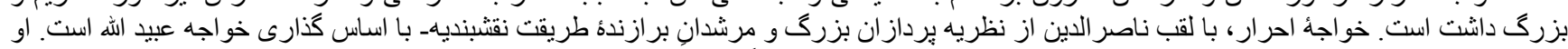

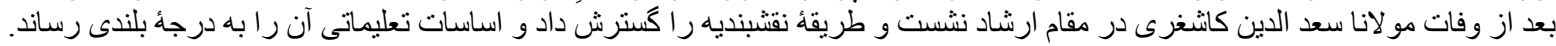

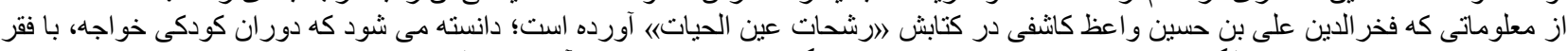

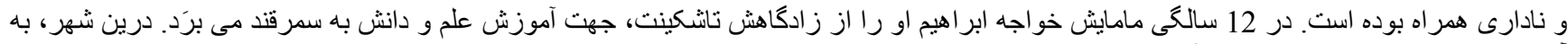

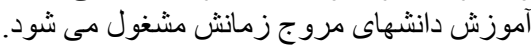

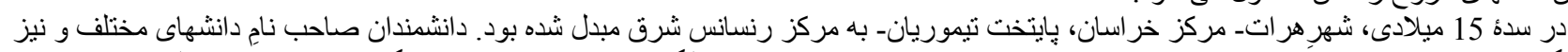

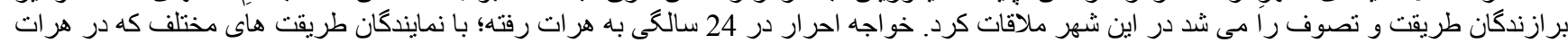

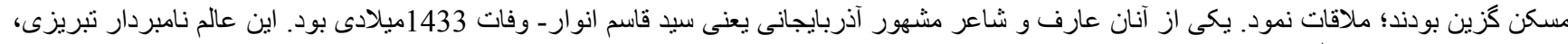

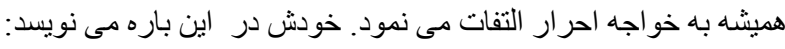

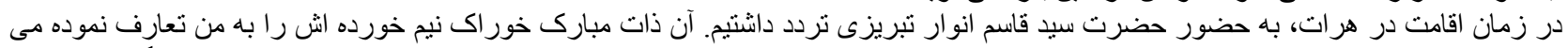

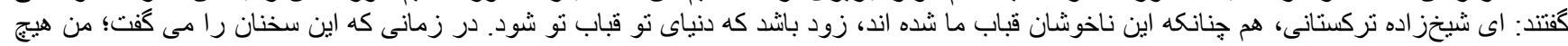

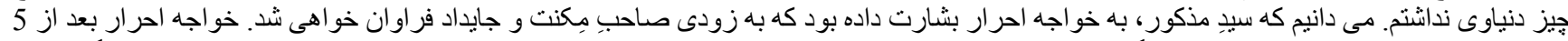

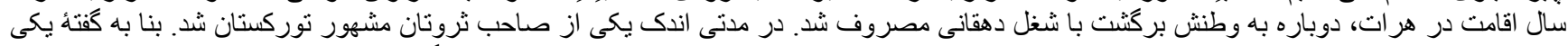

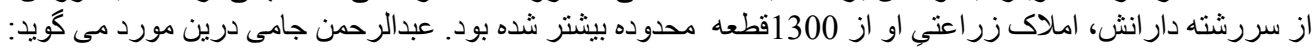

$$
\text { كز ارش مزر رفته رد در زير كثت است است }
$$

به همين گونه حضرت نوايى در منظومهُ 》احيرت الابرار) اشاره بيى به ثروتِ بيكران او دارد:

ملك جهان مزرعى، دهقانى اول

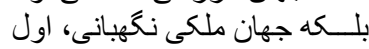

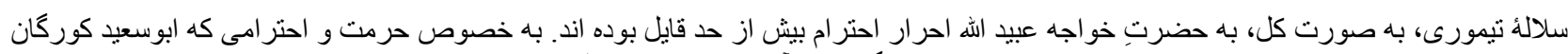

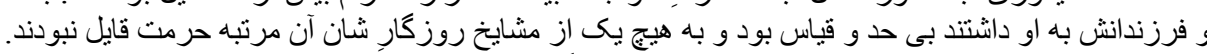

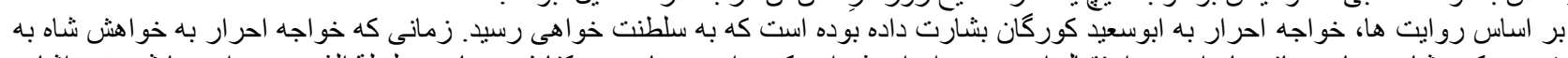

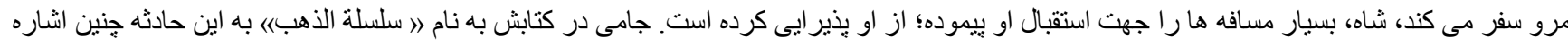

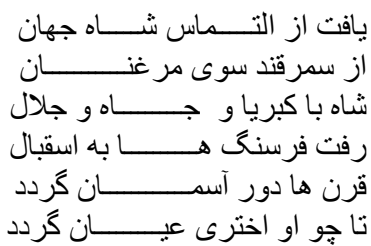

9.

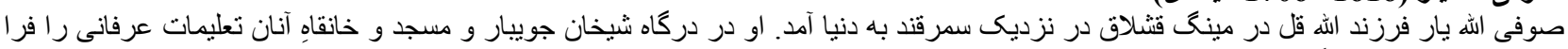

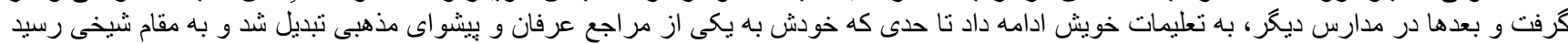

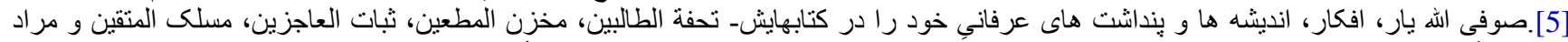

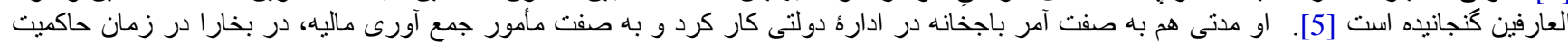

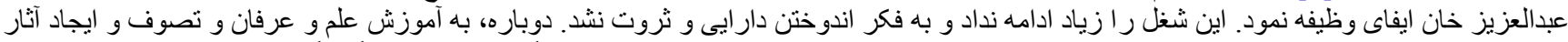

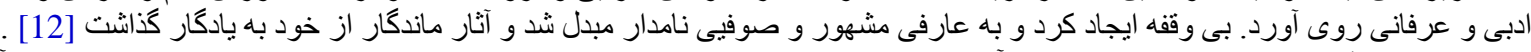

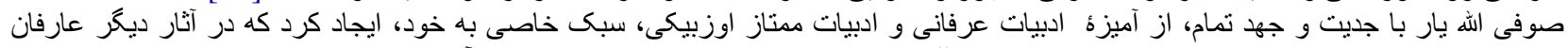

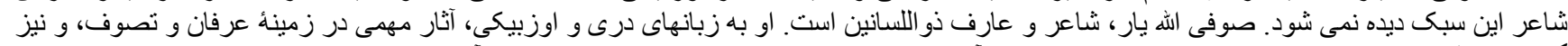

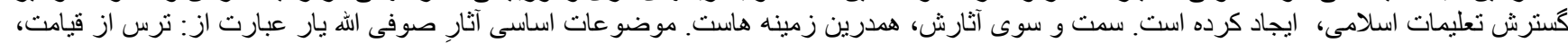




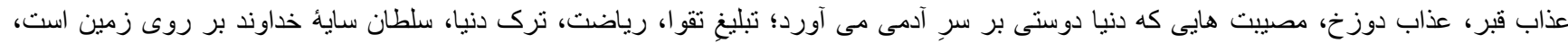

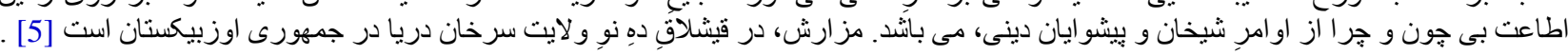

10

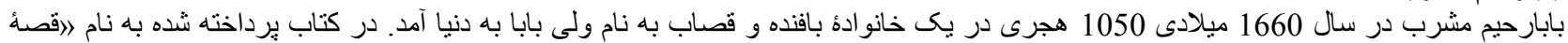

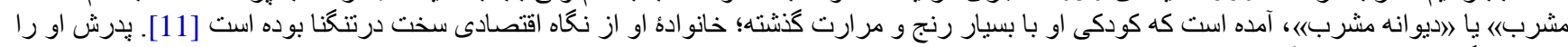

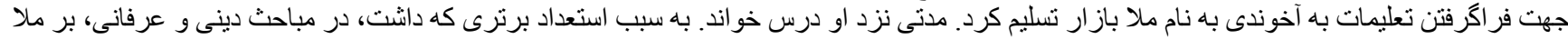

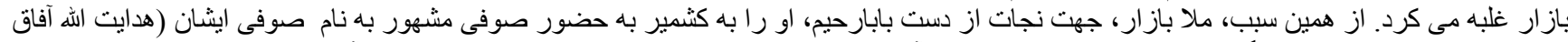

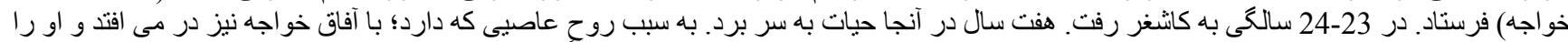

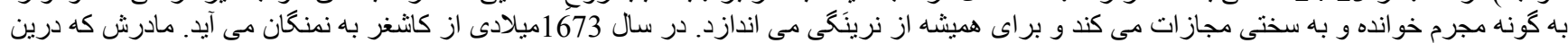

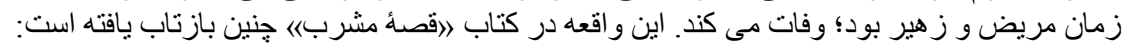

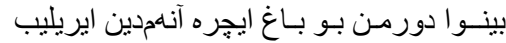

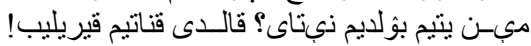

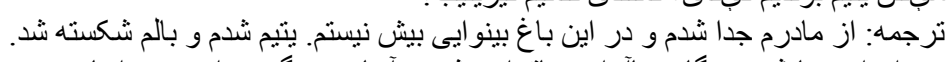

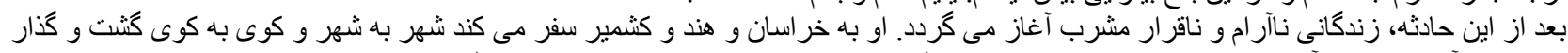

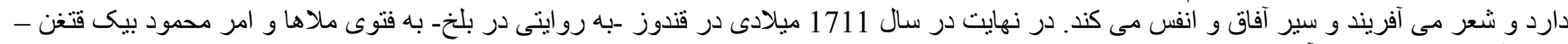

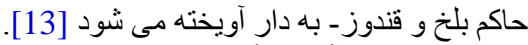

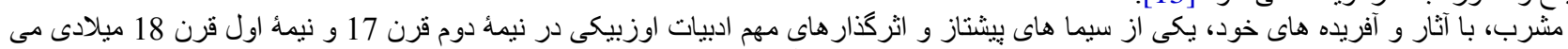

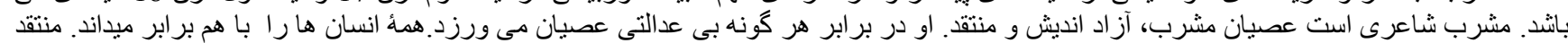

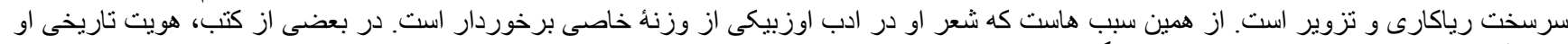

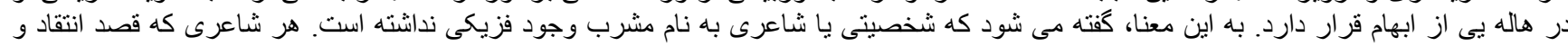

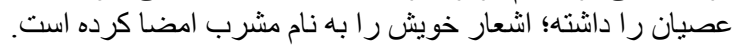

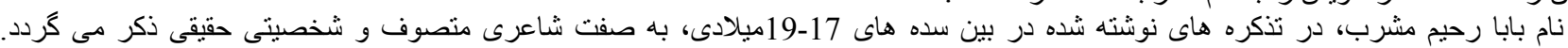

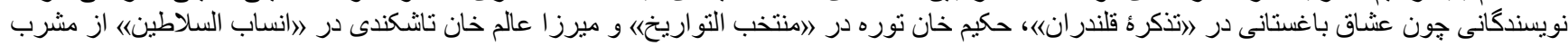
يادكرد هايى دارند.

\section{جهان بيني مشرب}

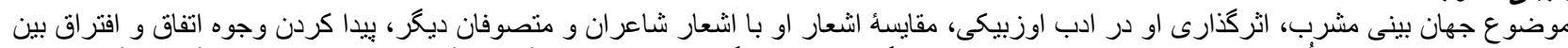

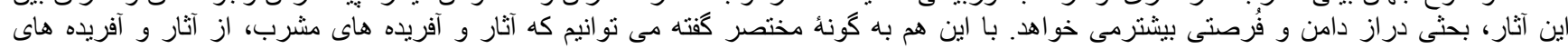

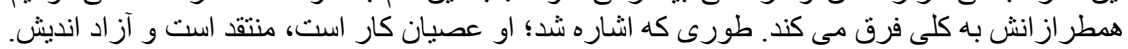

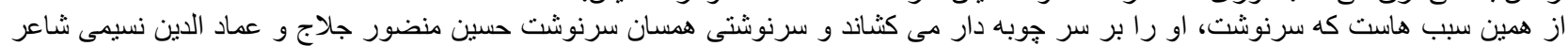

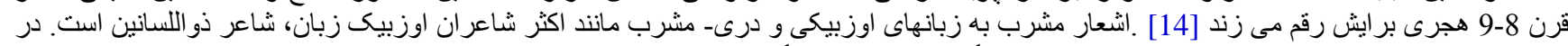

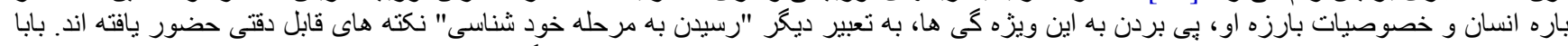

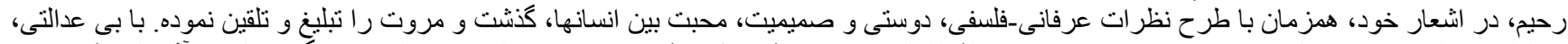

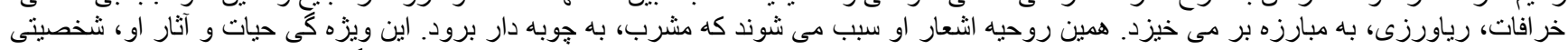

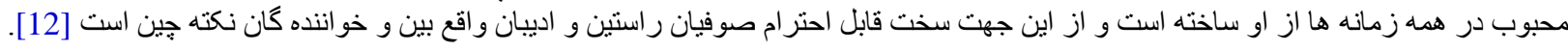

11. نتيجه كيرى

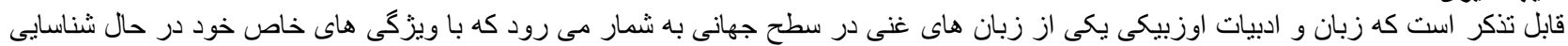

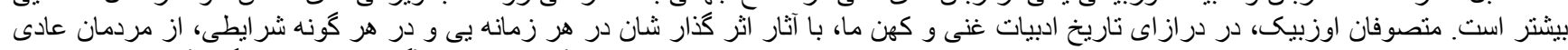

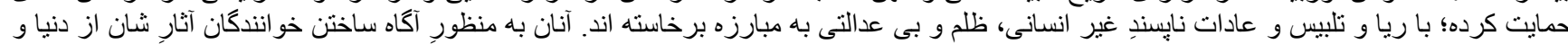

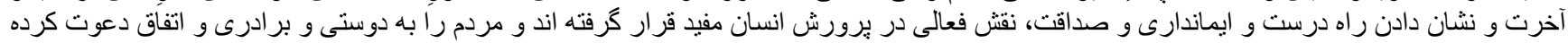

\section{References}

[1] S. Aminyar, "Uzbek historical literature," Kabul, Afghanistan, 1394.

[2] Al-Kashgari, Turkish language court. Ankara: Turk Dil Qoromo, 2015.

[3] Uzbekistan National Encyclopedia C, National encyclopedia of Uzbekistan. Tashkent: Authors, 2000.

[4] Z. Ishanch and M. Cocaine, Uzbek cultural literature. Faryab: Faryab State of Education, 1374.

[5] B. Toureyev, "Buyuk Yurt Allameh Lori (Signs of the Great Land)," Tashkent, Uzbekistan, 2018.

[6] B. J. Tukhlyov and B. Ahmadwa, "Literature collection 1," Tashkent Bayaz, 2018.

[7] B. J. Tukhlyov and B. Ahmadwa, "Literature collection 2," Tashkent Bayad, 2018.

[8] K. Y. H. Zumradi, "Critical review of the situation and works and narrations," vol. 14, pp. 55-54, 1385.

[9] S. Sajjadi, Introduction to the foundations of mysticism and Sufism. Tehran: Samat Publications, 1385.

[10] S. Jozjani, Sufism and man. Kabul: Saeed Publications, 2012.

[11] A. Ishrat, Sufism and sufi terms. Kabul: Al-Azhar Publications, 1391.

[12] Alishir Navai Dictionary Dictionary, Authors group. Tashkent: East of Publications, 2016.

[13] B. Bar and L. Start, Tuzla Tib Qat Publishing house of Mohammad Kazem Mini. Kabul: Al-Azhar Publishing House, 1394.

[14] Y. Mullah, Nettarikh of Uzbek Turkish literature vol. 1. Pakistan: Burhanuddin Namagh Shahrani, 1379. 